\title{
Several inverse problems in thermal physics
}

\author{
Ilmars Iltins \\ Department of Engineering Mathematics \\ Riga Technical University \\ Riga, Latvia \\ iltins@inbox.lv
}

\author{
Marija Iltina \\ Department of Engineering Mathematics \\ Riga Technical University \\ Riga, Latvia \\ marijai@inbox.lv
}

\begin{abstract}
There are solution approaches for several thermalphysical inverse problems discussed in the paper that are based on extension of non-stationery temperature field in the series. An applied method for separation of variables differs from the conventional method for separation of variables. Effect thereof differs as well and is especially convenient for solving inverse thermal-physical problems. Abraham Temkin (1919-2007) created this method for separation of variables. He also offered several methods for solving inverse problems. Those methods are not studied enough and are not known among a wider range of experts. Those methods have been created between 1956 and 1973 and have been published in various journals in Samara, Moscow, Minsk, and Riga. All those publications are in Russian and not available in electronic format.
\end{abstract}

Keywords - temperature field; inverse problem; series; temperature conductivity; boundary conditions.

\section{INTRODUCTION}

The first publication that we know and which laid the foundation for unconventional separation of variables is [1]. In that paper it is proved that a function that is defined by a convolution integral

$$
f(t)=\int_{a}^{t} \varphi(\tau) \xi(a+t-\tau) d(\tau),
$$

can be expanded in a following series

$$
f(t)=\sum_{k=0}^{\infty} \xi^{(k)}(a) \varphi^{(-k-1)}(t)
$$

where $\xi^{(k)}(a)$ is k-th derivative of function $\xi(t)$ at point a, $\xi^{(0)}(a)=\xi(a)$ and

$$
\varphi^{(-k)}(t)=\int_{a}^{t} \varphi^{(-k+1)}(\tau) d \tau, k=1,2,3, \ldots .
$$

The aforesaid paper illustrates that the series (2) converges if all derivatives $\xi^{(k)}(a)$ and all integrals $\varphi^{(-k)}(t)$ are limited. A. Temkin called that series (2) as a generalized Taylor series due to the fact that if the function

$$
\varphi(t)=\delta(t-a)
$$

where $\delta$ is the Dirac delta function, then

$$
\varphi^{(-k-1)}(t)=\frac{(t-a)^{k}}{k !}
$$

and in such a case the series (2) is an extension of function $f(t)$ in the Taylor series.

As solution of a heat transfer equation is expressed by a convolution integral, if initial distribution of temperature is homogenous and time-dependent boundary conditions are set on the boundary, it is obvious that attempt to apply the discussed series for solving a heat transfer equation is useful. Obtained solutions [2] are in the form convenient for one to apply them for solving inverse heat transfer problems. Later on A.Temkin had many publications devoted to those issues, whose results are summarized in a monograph [2] published in 1973. It should be noted that computing opportunities were quite limited at the time when those methods were created; therefore the methods have not been verified sufficiently.

\section{APPLICATION OF A GENERALIZED TAYLOR SERIES FOR SOLUTION OF A HEAT TRANSFER EQUATION}

A solution of heat transfer equation put down by means of a generalized Taylor series in a voluntary curved area is given in [2]. For the purpose of simplicity, we shall consider only the simplest segments where every point depends only on one coordinate of an area - a plate, a cylinder, and a sphere. If speaking on inverse heat transfer problems, solids of such particular kind are used for determination of thermo-physical properties of material. A symmetric temperature field in those solids is described by an equation

$$
\frac{\partial t}{\partial \tau}=a\left(\frac{\partial^{2} t}{\partial x^{2}}+\frac{k-1}{x} \frac{\partial t}{\partial x}\right)
$$

where $t$ is temperature, $\tau$ is time, $x$ is the co-ordinate, $k=1$ is the plate, $k=2$ is the cylinder, $k=3$ is the sphere, $a$-temperature conductivity coefficient. If convective heat exchange occurs with the environment having time-dependent temperature $t_{e}(\tau)$ on the surface, then following equality is valid on the boundary

$$
\lambda \frac{\partial t(b, \tau)}{\partial \tau}=\alpha\left(t_{e}(\tau)-t(b, \tau)\right),
$$


where $\lambda$ stands for heat transfer coefficient, $\alpha$ is heat exchange coefficient, $b$ is a half of thickness in case of a plate or radius in case of a cylinder or a sphere.

Initial conditions are

$$
t(x, 0)=t_{0} \text {. }
$$

When considering solution of problem (6) - (8), transition to dimensionless values is more convenient: $N=\frac{x}{b}$ is dimensionless co-ordinate $N \in[-1 ; 1]$ in case of a plate and $N \in[0 ; 1]$ in case of a sphere and a cylinder, $F=\frac{a \tau}{b^{2}}$ is dimensionless time, $B=\frac{\alpha b}{\lambda}$ is dimensionless heat exchange coefficient, $T=\frac{t(x, \tau)-t_{0}}{t_{e}^{*}-t_{0}}$ is dimensionless temperature, $t_{e}^{*}=\max _{\tau \geq 0} t_{e}(\tau)$. Problem (6) - (8) in dimensionless form shall be written down as follows

$$
\begin{aligned}
& \frac{\partial T}{\partial F}=\frac{\partial^{2} T}{\partial N^{2}}+\frac{k-1}{N} \frac{\partial T}{\partial N} \\
& \frac{\partial T(1, F)}{\partial N}=B\left(T_{e}(F)-T(1, F)\right) \\
& T(N, 0)=0
\end{aligned}
$$

According to [2], solution of the problem (9) - (11) is of the following form, where

$$
\begin{aligned}
& T_{1}(N, F)=\sum_{n=0}^{\infty} T_{e}^{(n)}(F) P_{n}(N) \\
& T_{2}(N, F)=\sum_{n=0}^{\infty} T_{e}{ }^{(n)}(0) P_{n}(N, F)
\end{aligned}
$$

Function $T_{1}(N, F)$ satisfies equation (9) and boundary conditions (10), whereas function $T_{2}(N, F)$ satisfies equation (9) and those boundary conditions

$$
\frac{\partial T_{2}(1, F)}{\partial N}+B T_{2}(1, F)=0
$$

This result is obtained in two ways in the [2], id est, by inserting (13) and (14) in the equation (9) and equalling the coefficients to derivatives of the same order $T_{e}{ }^{(n)}(F)$ and to the latter from the conventional model.

Here it should be noted that solution of the problem (9) is also expressed in the form of (12) - (14) under boundary conditions of different type. Functions $P_{n}(N)$ depend on a type of boundary conditions and geometry of an area. Those are either polynomials or functions where a polynomial is included as addend. In this account those functions are called quasi polynomials in the [2].

For initial condition (11) be met, the following is set in formula (14)

$$
P_{n}(N, F)=-P_{n}(N) \text {. }
$$

There is proved in the [2] that $\lim _{F \rightarrow \infty} T_{2}(N, F)=0$, thus at large values $F$ addend $T_{2}(N, F)$ can be not taken into account in formula (12). The following inequality shows that [2]

$\left|P_{n}(N, F)\right|<\left(\frac{1}{2 k}\right)^{n} e^{-\mu F}$,

where $\mu>0$.

It is proved in the [2] that $P_{2 n}(N)>0, P_{2 n+l}(N) \leq 0$ and that inequality is valid

$$
\left|P_{n}(N)\right| \leq\left(\frac{1}{k B}+\frac{1}{2 k}\right)^{n} \text {. }
$$

Therefore if $B>1$ and all derivatives $T_{e}{ }^{(n)}(F)$ are limited, then (13) converges. Besides the more intense heat exchange between the solid and the ambience, the faster the series converges.

There is method for determination of an asymmetric temperature field discussed in the [2].

\section{DETERMINATION OF TEMPERATURE CONDUCTIVITY COEFFICIENT}

Temperature conductivity coefficient is determined in laboratory conditions, by measuring temperature inside a simple-shaped solid while it is warmed up (cooled down more rarely). Heat transfer process is described by an equation (6), where $x \in[0, b]$. Temperature is measured at two inner points $x_{1}$ and $x_{2}, x_{1}<x_{2}$. There is a case possible when $x_{2}=b$. Supposing that $x_{2}=b$ and transferring to dimensionless values as showed before, but keeping real temperature in equation (9), we get that heat transfer process can be described by equation (9) and boundary conditions

$$
t(1, F)=t_{1}(F), \quad \frac{\partial t(0, F)}{\partial N}=0,
$$

where $t_{l}(F)$ is temperature measured on the boundary $x=b$. Solution of problem (9), (19) at sufficiently high $F$ values is written down as follows [2]

$$
t(N, F)=\sum_{n=0}^{\infty} P_{n}(N) t_{1}{ }^{(n)}(F)
$$

Co-ordinate functions $P_{n}(N)$ are given in the [2]:

if $k=1$, then

$P_{0}(N)=1$, 


$$
\begin{aligned}
& P_{1}(N)=\frac{N^{2}}{2}-\frac{1}{2}, \\
& P_{2}(N)=\frac{N^{4}}{24}-\frac{N^{2}}{4}+\frac{5}{24}, \\
& P_{3}(N)=\frac{N^{6}}{720}-\frac{N^{4}}{48}+\frac{5 N^{2}}{48}-\frac{61}{720}, \\
& P_{4}(N)=\frac{N^{8}}{40320}-\frac{N^{6}}{1440}+\frac{5 N^{4}}{576}-\frac{61 N^{2}}{1440}+\frac{277}{8064} . \\
& \text { If } k=2, \text { then } \\
& P_{0}(N)=1, \\
& P_{1}(N)=\frac{N^{2}}{4}-\frac{1}{4}, \quad N^{4} \\
& P_{2}(N)=\frac{N^{2}}{64}+\frac{3}{16}, \\
& P_{3}(N)=\frac{N^{6}}{2304}-\frac{N^{4}}{256}+\frac{3 N^{2}}{256}-\frac{19}{2304}, \\
& P_{4}(N)=\frac{N^{8}}{147456}-\frac{N^{6}}{9216}+\frac{3 N^{4}}{4096}-\frac{19 N^{2}}{9216}+\frac{211}{147456} .
\end{aligned}
$$

When transferring from dimensionless time $F$ to real time $\tau$ in formula (20), we get

$$
t(N, \tau)=\sum_{n=0}^{\infty} P_{n}(N) t_{1}{ }^{(n)}(\tau)\left(\frac{b^{2}}{a}\right)^{n} .
$$

Temperature at $N_{l}=x_{1} / b, N_{l} \in[0,1]$ is measured. Thus, taking final number of addends in formula (21) and denoting $b^{2} / a=y$, we obtain

$$
\sum_{n=1}^{M} P_{n}\left(N_{1}\right) t_{1}^{(n)}(\tau) y^{n}+t_{1}(\tau)-t\left(N_{1}, \tau\right)=0,
$$

Because $P_{0}(N)=1$ for all $k=1,2,3$ [2]. In this way we get that $y$ is a polynomial root. If $M=1$, then it follows from (22) that

$$
a=\frac{b^{2} P_{1}\left(N_{1}\right) t_{1}^{\prime}(\tau)}{t\left(N_{1}, \tau\right)-t_{1}(\tau)} .
$$

If temperature is measured in the middle $\left(N_{l}=0\right)$, then if $k=1$, it results from (23) that

$$
a=\frac{b^{2} t_{1}^{\prime}(\tau)}{2\left(t_{1}(\tau)-t(0, \tau)\right)},
$$

and if $k=2$, then

$$
a=\frac{b^{2} t_{1}^{\prime}(\tau)}{4\left(t_{1}(\tau)-t(0, \tau)\right)} .
$$

Formulas (23) - (25) are found in the [2] by means of an approach described in this article. The [3] provided formulas (24) and (25), where they are obtained in a different way.
It is clear that a more precise result is anticipated if several addends are taken in the sum (22). If $M=2$, then it follows from (22) that

$$
P_{2}\left(N_{1}\right) t_{1}^{\prime \prime}(\tau) y^{2}+P_{1}\left(N_{1}\right) t_{1}^{\prime}(\tau) y+t_{1}(\tau)-t(N, \tau)=0
$$

Equation (26) is a quadratic equation, thus it has two roots wherefrom only one is valid. There is justified in the [4] that if $t_{1}{ }^{\prime}(\tau)>0$ and $t_{1}{ }^{\prime \prime}(\tau)<0$ that is not hard to be ensured experimentally, multiplication of the roots from equation (26) is negative. Hence a question as regards a valid root fools away, because $y$ must be definitely positive.

If temperature is measured in the centre $\left(N_{1}=0\right)$ and $k=1$, then (26) results in [2], [4]

$$
\frac{5}{24} t_{1}^{\prime \prime}(\tau) y^{2}-\frac{1}{2} t_{1}^{\prime}(\tau) y+t_{1}(\tau)-t_{1}(0, \tau)=0 \text {. }
$$

When $M=3, N_{l}=0$ and $k=1$, then the following occurs [4]:

$$
\begin{aligned}
& -\frac{61}{720} t_{1}{ }^{(3)}(\tau) y^{3}+\frac{5}{24} t_{1}{ }^{\prime \prime}(\tau) y^{2}- \\
& -\frac{1}{2} t_{1}{ }^{\prime}(\tau) y+t_{1}(\tau)-t_{1}(0, \tau)=0
\end{aligned} .
$$

In the [4] we referred to a large number of calculations made, by using mathematical software, which illustrated that equation (28) had one real and two complex roots at most various boundary conditions $t_{l}(\tau)$. The [4] compares accuracy of formulas (24), (27), and (28) for determination of temperature conductivity coefficient with temperature field being chosen as input data, which is obtained at a given temperature conductivity coefficient, by solving a heat transfer equation numerically by means of mathematical software. It is concluded that formula (27) and (28) is significantly more precise than formula (24) and that accuracy of all discussed formulas improves if time is increased while temperatures used in calculations are recorded.

\section{CALCULATION OF BOUNDARY CONDITIONS AS PER TEMPERATURE MEASUREMENTS INSIDE A SOLID}

Surface temperature of a solid can be determined by technical means (thermal imaging camera), but it requires a surface be open and accessible. If it is not the case, thermal imaging camera cannot be applied. Temperature measurements inside a solid by means of a thermocouple, and determination of surface temperature of a solid by using those measurements would be a solution. Such approach is recommended in the [2].

Let us consider simple-shaped solids only where heat transfer process may be described by equation (6) and $x \in[0, b]$. it should be noted that this approach may be also applied to solids of complex shapes. Thermal-physical properties of material that is heat transfer coefficient $\lambda$ and temperature conductivity coefficient $a$ are known. Let us suppose that temperature is measured at an inner point of area $x_{1}=b_{1}, b_{1}<b$. Then in area $x \in\left[0 ; b_{1}\right]$ equation (6) with the following boundary conditions is valid

$$
t\left(b_{1}, \tau\right)=t_{1}(\tau)
$$


where $t_{l}(\tau)$ is the measured temperature. Temperature field in the area $x \in\left[0 ; b_{I}\right]$ can be calculated unequivocally. Temperature $t(b, \tau)$ must be found. The area $x \in\left[b_{1}, b\right]$ is a plate $(k=1)$, an empty cylinder $(k=2)$ or an empty sphere $(k=3)$ with thickness $b-b_{l}$. If we could determine temperature field in this area, temperature $t(b, \tau)$ would be also known. So that temperature field in this area would be determined unequivocally, boundary conditions must be set on both boundaries of the area. But it is impossible to set boundary condition at $x=b$, because it is a calculated value. It is known according to [5] that temperature field in solids of such type is calculated unequivocally if boundary conditions of two different types are set on the boundary $x=b_{1}$ while boundary conditions on the boundary $x=b$ are not set. In the [2] boundary conditions of such kind are called boundary conditions of the fourth type named after Likov. As temperature field in the area $\left[0, b_{1}\right]$ is calculated, then heat flow is also calculated at $x=b_{1}$, namely, $q(\tau)=\lambda \frac{\partial t(b, \tau)}{\partial x}$. Therefore in the area $\left[b_{1}, b\right]$ equation

(6) with boundary conditions (29) is valid, and

$$
\lambda \frac{\partial t(b, \tau)}{\partial x}=q(\tau)
$$

where $q(\tau)$ is got from solution of problem (6), (29). When problem (6), (29), and (30) is transferred to dimensionless form, we get equation (9), where $N=x / b_{1}, N \in\left[1, b / b_{1}\right]$, and boundary conditions are

$$
\begin{aligned}
& T(1, F)=T_{1}(F) \\
& \frac{\partial T(1, F)}{\partial N}=U(F)
\end{aligned}
$$

In the last formula $U(F)$ is acquired from solution of problem (9), (31). Solution of problem (9), (31), and (32) in the case of high $F$ values is searched in the following form [6]:

$$
T(N, F)=\sum_{n=0}^{\infty} U^{(n)}(F) P_{n}(N, 1)+\sum_{n=0}^{\infty} T_{1}^{(n)}(F) P_{n}(N, 2)
$$

where co-ordinate functions $P_{n}(N, 1)$ and $P_{n}(N, 2)$ are searched, by inserting (33) in equation (9) and requesting compliance with the boundary conditions. The solution of described problem if $k=2$ can be written down as a recurrent formula

$$
\begin{gathered}
P_{n+1}(N, m)=\int_{1}^{N} \frac{d \eta}{\eta} \int_{1}^{\eta} \xi \cdot P_{n}(\xi, m) d \xi, m=1,2 \\
P_{0}(N, 1)=-\ln N, \quad P_{0}(N, 2)=1
\end{gathered}
$$

Interesting to note that in the [2] solution of problem (9), (31), and (32) is not obtained in the form (34), (35). Author of the [2] followed the following scheme. Solution of problem (9), (31) at high $F$ values and $N \in[0,1]$ have the form

$$
T(N, F)=\sum_{n=0}^{\infty} T_{1}^{(n)}(F) P_{n}(N)
$$

Thus,

$$
U(F)=\sum_{n=0}^{\infty} T_{1}^{(n)}(F) P_{n}^{\prime}(1) .
$$

Ultimately both series are merged in one ultimately in formula (33), whose each addend is multiplication of temperature $T_{l}(F)$ derivatives and corresponding co-ordinate functions. Hence, solution of problem (9), (31), and (33) is found in the form (36). It is proved in the [2] that solution of problem (9), (31) if $N \in[0,1]$, and (9), (31), and (32) if $N>1$ is (36), as well as coordinate functions $P_{n}(N)$ are the same in both problems unless $U(F)$ in boundary condition (32) is set by formula (37). Eventually temperature on the area boundary is calculated according to formula

$$
T(b, F)=\sum_{n=0}^{\infty} T_{1}^{(n)}(F) P_{n}\left(\frac{b}{x_{1}}\right) .
$$

\section{DETERMINATION OF TEMPERATURE CONDUCTIVITY COEFFICIENT FOR THIN MATERIALS}

If material is thin, for instance, window glass, film, etc., thermocouple cannot be placed inside such material. In such a case the studied material can be placed between two materials with known thermal-physical properties and thermocouples can be placed in these materials as showed in figure 1 . In such a scheme thermocouple co-ordinates are $x_{1}, x_{2}, x_{3}$, and $x_{4}$. Temperature conductivity coefficient $a_{l}$ and heat transfer coefficient $\lambda_{1}$ are established. The studied material is located between $x=0$ and $x=b$.

In order to determine temperature conductivity coefficient $a$ of the studied material, the following scheme is proposed in the [2]:

1. Heat flow $q\left(x_{2}, \tau\right)$ at point $x=x_{2}$ is established according to temperature measurements at points $x=x_{1}$ un $x=x_{2}$.

2. When $t\left(x_{2}, \tau\right)$ and $q\left(x_{2}, \tau\right)$ are known, $t(0, \tau)$ and $q(0, \tau)$ are calculated as it is shown in the previous paragraph.

3. Having applied temperature measurements at points $x=x_{3}$ and $x=x_{4}, t(b, \tau)$ and $q(b, \tau)$ are calculated similarly.

4. Ratio $q(b, \tau) / q(0, \tau)$ can be calculated - that is a value obtained experimentally.

5. According to known $t(0, \tau)$ and $t(b, \tau)$, temperature field at range $x \in[0, b]$ is found, and ratio $q(b, \tau) / q(0, \tau)$ is calculated therefrom. This ratio depends on wanted temperature conductivity coefficient $a$.

6. Having equalled results obtained pursuant to point 4 and 5 above, temperature conductivity coefficient $a$ is established.

One should note that it is important so that $t(-l, \tau)$ would not be equal to $t(l+b, \tau)$. 


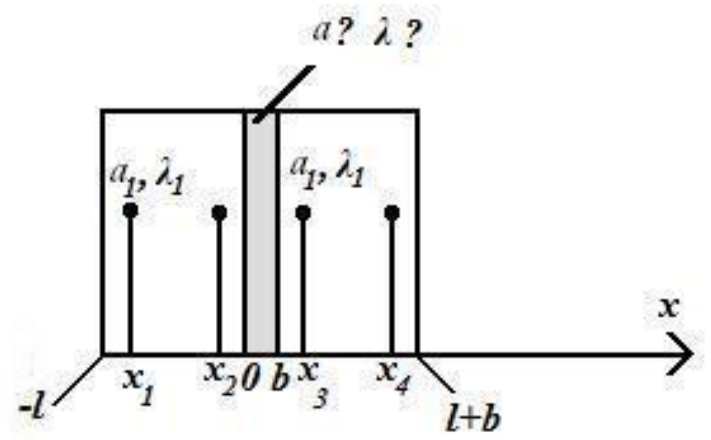

Fig. 1 Detection scheme of the heat physical characteristics.

The discussed scheme is implemented in [7], by using temperature field in the area $x \in[-l, l+b]$ as input data that is calculated by means of mathematical software MATHEMATICA, and by solving the problem numerically. The [7] includes a conclusion that the scheme described in the [2] runs, but restrictions as regards thickness $b$ of studied material exist. If $b$ is decreased, then problem becomes ill conditioned and it is impossible to establish temperature conductivity coefficient. It manifests itself in such a way that time range $\left[\tau_{1}, \tau_{2}\right]$ does not exist at too small $b$ values where $a$ is constant. Physically it means that if thickness of the studied material is too little, then this material does not practically affect readings of thermocouple if compared to the case if the studied material would not exist at all.

\section{CONCLUSIONS}

The examined solution of heat transfer equation and inverse problems associated therewith are less known to a wide range of experts. Practical calculations given in [4], [6], [7] illustrates that theoretical concepts summarized in the [2] are applicable for solving inverse heat transfer equations. Discussed problems are only a part of scientific heritage left by A.Temkin. Major part is not studied yet up to date.

\section{REFERENCES}

[1] A.Temkin, "Generalised Taylor series and the theorem of product of images," Kuibishev Industrial Institute, Kuibishev, pp. 529-551, 1956. (in Russian)

[2] A. Temkin, "Inverse methods of thermal conductivity", Moscow: Energya, 1973. (in Russian)

[3] A. Sashov, G. Volohov, T. Abramenko and V. Kozlov, "Methods of determination of heat and temperature transfer," Moscow: Energya, 1973. (in Russian)

[4] I. Iltins and M. Iltina, "Determination of heat physical characteristics by using series along boundary condition derivatives", WSEAS Press, Proceedings of the $2^{\text {nd }}$ International Conference on Mathematical Models for Engineering Science. Puerto De La Cruz, Tenerife, Spain, December 10-12, 2011.

[5] A. Likov, "Heat and mass transfer," Moscow: Energya, 1978. (in Russian)

[6] I. Iltins, "A. Temkin's method of separation of variables", Scientific Proceedings of Riga Technical University, Computer Science, vol. 25, pp. 55-61, 2005.
[7] I. Iltins and M. Iltina, "One method of determination of thermal and physical characteristics of film-base materials," Scientific Proceedings of Riga Technical University, Computer Science, vol. 50, pp. 67-71, 2011.

\section{Creative Commons Attribution License 4.0 (Attribution 4.0 International, CC BY 4.0)}

This article is published under the terms of the Creative Commons Attribution License 4.0 https://creativecommons.org/licenses/by/4.0/deed.en_US 